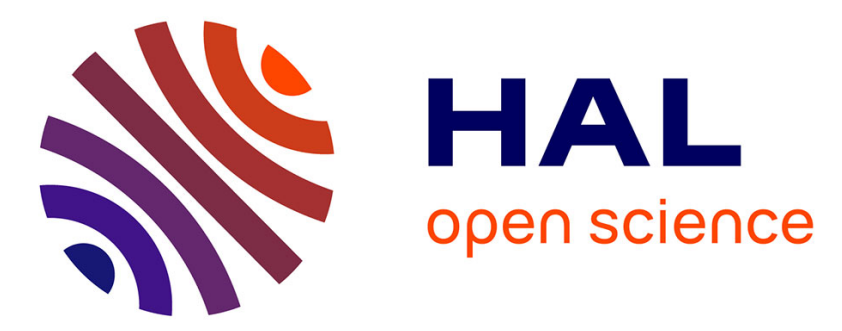

\title{
Tuning the ordered states of folded rods by isotropic confinement
}

\author{
Elsa Bayart, A. Boudaoud, Mohktar Adda-Bedia
}

\section{To cite this version:}

Elsa Bayart, A. Boudaoud, Mohktar Adda-Bedia. Tuning the ordered states of folded rods by isotropic confinement. Physical Review E: Statistical, Nonlinear, and Soft Matter Physics, 2014, 89 (1), 10.1103/physreve.89.012407 . hal-03025059

\section{HAL Id: hal-03025059 \\ https://hal.science/hal-03025059}

Submitted on 26 Nov 2020

HAL is a multi-disciplinary open access archive for the deposit and dissemination of scientific research documents, whether they are published or not. The documents may come from teaching and research institutions in France or abroad, or from public or private research centers.
L'archive ouverte pluridisciplinaire HAL, est destinée au dépôt et à la diffusion de documents scientifiques de niveau recherche, publiés ou non, émanant des établissements d'enseignement et de recherche français ou étrangers, des laboratoires publics ou privés. 


\title{
Tuning the ordered states of folded rods by isotropic confinement
}

\author{
E. Bayart, ${ }^{1}$ A. Boudaoud, ${ }^{2}$ and M. Adda-Bedia ${ }^{1}$ \\ ${ }^{1}$ Laboratoire de Physique Statistique, Ecole Normale Supérieure, UPMC Paris 6, \\ Université Paris Diderot, CNRS, 24 rue Lhomond, 75005 Paris, France \\ ${ }^{2}$ RDP, ENS Lyon, 46 allée d'Italie, 69007 Lyon, France
}

(Received 29 July 2013; published 23 January 2014)

\begin{abstract}
The packing of elastic objects is increasingly studied in the framework of out-of-equilibrium statistical mechanics and thus these appear to be similar to glassy systems. Here, we present a two-dimensional experiment whereby a rod is confined by a parabolic potential. The setup enables spanning a wide range of folded configurations of the rod. Measurements of the distributions of length and curvature in the system reveal the importance of a stacking process whereby many layers of the rod are grouped into branches. The geometrical order of patterns increases with the confinement strength. Measurements of the distributions of energies lead to the definition of an energy scale that is correlated with the elastic energy of the stacked parts of the rod. This scale imposes energy partition in the system and might be relevant to the framework of the thermodynamics of disordered systems. Following these observations, we describe the patterns as excited states of a ground state corresponding to the most ordered geometry. Eventually, we provide evidence that the disordered state of a folded rod becomes spontaneously closer to the ground state as confinement is increased.
\end{abstract}

DOI: 10.1103/PhysRevE.89.012407

PACS number(s): 46.32.+x, 46.70.Hg, 61.43.-j

\section{INTRODUCTION}

Confined elastic structures such as plates and rods are ubiquitous in natural phenomena and man-made systems. The length scales associated with the underlying folding, wrinkling, and crumpling processes range from microscopic to macroscopic sizes. One influential example concerns the behavior of balls of polymer chains and their complex geometry in the case of poor-solvent interactions [1]. In biological systems, how elastic structures adapt themselves to fit in a small container is of paramount importance concerning nucleic polymers (DNA, RNA). In the cell nucleus, DNA is embedded within a fiberlike structure known as chromatin, which seems to be packed in disordered patterns with multiscale features that might be relevant to transcriptional regulation. In contrast, in viruses, DNA or RNA filaments are perfectly ordered when confined into the viral capsid by a molecular motor which is essential for proper function [2,3]. At larger scales, it is common for living tissues to grow within the walls of a protective, stiffer, surrounding medium. A salient example is the growth of tree leaves inside a bud; the geometrical frustration resulting from such a constrained development affects the final shape of the leaves and the organization of their veins $[4,5]$. This raises the question of interaction between mechanical stress and molecular biology in the context of biological growth. Finally, the confinement of elastic objects is also linked to technological challenges. A better understanding of the fundamental physical principles governing the wrinkling of elastic plates would lead to smarter stretchable electronic devices [6], which, for instance, would be mounted on living tissues so as to prevent body rejection. Another aim concerns the production of self-deployable devices such as solar wings [7] through the reversible folding of ordered and disordered patterns without the risk of tearing the structure.

The few examples mentioned above make it clear that a plethora of complex fold patterns are displayed by tightly packed elastic structures across a wide spectrum of natural phenomena as well as in many man-made systems. Questions about the configurations and physical properties of folded and packed elastic structures have recently begun to catch the attention of a growing number of groups across the soft-matter community. An important reason for this development is that packings, like frustrated and disordered systems such as glasses, are increasingly being looked at through the prism of "out-of-equilibrium" statistical mechanics. Because of the macroscopic size of the physical systems typically under study (ranging from granular packings to elastic plates and even liquid foams), thermal fluctuations are completely negligible with respect to other forces, such as gravity, and play no role in the respective phase space exploration of these athermal systems. Rather, one must resort to mechanical excitations (for example the so-called tapping in the case of granular matter) in order to drive the system from one local energy minimum to another one. One of the most interesting questions that has emerged in recent years concerns the possibility of defining a new form of thermodynamics that would describe such disordered athermal systems.

Despite its mundane appearance and usually undistinguished presence, we propose that a piece of crumpled paper actually represents a prototype in which this new thermodynamics of disordered systems can be studied. When a sheet of paper is crushed into a small ball, the manual energy (injected at scales comparable to the size of the sheet itself) localizes into a complex network of linear singular folds connected to each other via pointlike singular structures. Although the mechanical and geometrical properties of these two individual singular components have been the subject of intense study in the past few years [8-14], the dynamical formation of the network connecting them (the skeleton of a fully developed piece of crumpled paper) remains only very partially understood [15]. Besides the very first stages of the folding dynamics which can be followed with some accuracy using the equations of elasticity, the crumpled pattern rapidly becomes too complex and it is impossible to describe the whole evolution even through numerical methods [16,17]. One 
major hurdle comes from the nonlocal hard-core interactions due to the physical constraint of self-avoidance. Therefore, most studies have focused on the statistical properties and on the global mechanical response of balls of crumpled paper. Until recently, the usual technique to study crumpled paper was by unfolding the ball in order to measure some statistical distributions of its geometrical properties post mortem [18-20]. In this way a lot of information has been gained about the fractal coefficients of the resulting self-affine surface such as its roughness exponent [18]. However, it has now been demonstrated that one can gain full and nonintrusive access to the geometry of a folded ball by $\mathrm{x}$-ray tomography or numerical models, opening the door for even more precise measurement of the statistical geometry of crumpled paper [21-24]. Of principal interest is whether the hierarchical appearance of ridges and $d$ cones can be viewed as some form of energy cascade from large to small length scales resembling the scenario responsible for three-dimensional (3D) fluid turbulence [15].

In addition to its geometrical and mechanical properties, crumpled paper appears as an attractive system because it might be relevant to the framework of glassy systems. In this sense, the situation of crumpled paper mirrors that of early studies pointing towards a thermodynamic theory of granular matter. Just as in a regular granular pile, one can define a crystalline state for a confined sheet of paper where all the ridges are parallel to each other and large portions of the material are in self-contact [15]. However, without any special care during the folding process, one would expect to find the confined sheet in a rather disordered state. This can be viewed as a form of quenching [15]. In general, the folding pattern which is held in place by friction and self-avoidance cannot be changed by thermal fluctuations alone, and some sort of external forcing must be provided to relax this jammed system towards states of lower energy. In addition to this, it has been experimentally observed that the size of a crumpled ball of paper under a weight decreases logarithmically in time [25]. Even though the riddle about the exact reason (geometric rearrangements of the fold configuration or plastic creep in the ridges [26]) for this behavior is not yet fully resolved, the slow relaxation by itself is strikingly reminiscent of aging, thereby drawing further analogies to the framework of glassy systems.

All of the experimental features mentioned above make it very tempting to try to define a new form of thermodynamics essentially inspired by the early efforts of Edwards and Oakeshott in the context of granular matter [27]. In particular, concepts such as configurational entropy and effective temperatures are expected to play a prominent role [3]. Attempts to define such quantities have been made in the context of crumpled sheets [28-30]. However, the complexity of geometries and the difficulty of dealing with plastic deformations prevent grasping the essential features of such systems. Self-avoidance and internal friction are two main ingredients allowing the appearance of complexity as the available energy increases. Emergence of plastic deformations along ridges and $d$ cones are consequences of the pattern selection but do not take part in the primary process of selection. In this context, the confinement of a rod (1D) might a relevant model for the confinement of a sheet (2D). Investigations have been carried out in the context of an elastic rod in a
2D [16,28-30] or 3D container [31,32]. Despite promising results (the discovery of robust statistical distributions for example) and auspicious hints (the possible existence of a "thermalization" process between subsystems), the hunt for the underlying out-of-equilibrium theory of confined elastic rods remains embryonic [16,28-30]. In fact, those previous studies have so far been unable to draw conclusions about the precise mechanism(s) responsible for pattern selection in the folding process. Mainly, this is due to an inextricable experimental dilemma which has made it nearly impossible to study simultaneously the geometry and the mechanics of confined elastic rods without introducing some experimental biases such as friction with a rigid container, anisotropy of material injection, or material plasticity. These biases amount to an uncontrolled external forcing on the rod that might hide the elementary physics that we wish to uncover.

Recently, we set out to remove these limitations by designing an experimental system which allows isotropic and reversible confinement of an elastic rod without using any contact between the container and the periphery of the folded configuration [33]. The idea was to use a rotating Hele-Shaw cell in order to drive the rod inwards via the centripetal force and control the resulting confinement intensity by varying the stiffness of the underlying isotropic radial potential. This previous study enabled the characterization of the order of a folded rod, while the present study focuses on the dynamics of the emergence of order, and aims at proposing quantities that would be relevant for a thermodynamical theory. In our previous work, we were able to define the density of "geometrical order" of the folded configurations by measuring the number of parallel layers. Those results led us to speculate that a spiral-like pattern, where large portions of the rod are superposed on each other, constitutes the ground state of a confined rod in a plane. We proposed that this configuration is analogous to the crystalline state of minimal energy. In this article, we provide evidence suggesting not only that this conjecture is true but that the thermodynamical framework does in fact go beyond what has been anticipated so far. In particular, we demonstrate that typical disordered fold configurations can be understood as excited states with respect to the spiral ground state and that those states can relax towards their crystalline state. In order to establish this conclusion, the paper is organized as follows. First, we present the experimental setup. Then we provide statistical measurements of geometrical and mechanical quantities. Then we observe the existence of a stacking process, i.e., the superposition of layers of rods. Finally, we demonstrate the existence of an energy scale, imposed by the stacking process, which we believe to be a solid starting point upon which an out-of-equilibrium thermodynamical theory could be built.

\section{EXPERIMENTS}

The principle of the experiment has been explained in [33] and is reproduced in this section for completeness. An elastic rod is inserted into a circular Hele-Shaw cell that is filled with a liquid and entrained by a motor. The cell is slightly thicker than the rod, so that the rod cannot cross itself, constraining a two-dimensional folding. The liquid is denser than the rod. As a consequence, when the cell is rotated, the 
rod is subjected to a centripetal force and thus confined in a radial, parabolic pressure field $P(r)=P_{0}+\rho_{\ell} \omega^{2} r^{2} / 2$, where $r$ is the distance from the center of the cell, $\rho_{\ell}$ is the fluid mass density, $\omega$ is the angular velocity, and $P_{0}$ is the pressure at the center of the cell. We have checked that at the rotational velocities used, gravitational effects are negligible. The cell was fixed in a vertical position on the axis of a $2 \mathrm{~g} \mathrm{~cm}^{-3} \mathrm{~kW}$ motor, through a double ball bearing to avoid vibration transmission from the motor to the cell. The frequency of rotation $f=\omega / 2 \pi$ was controlled using an electronic frequency variator. The flexible rod used in all experiments is made of polydimethylsiloxane (Goodfellow) and has the following characteristics: diameter $d=(2 \pm 0.2) \mathrm{mm}$, total length $L=(3 \pm 0.01) \mathrm{m}$, mass density $\rho_{r}=1 \mathrm{~g} / \mathrm{cm}^{3}$, and Young's modulus $E=(1.0 \pm 0.1) \mathrm{MPa}$ (measured using a tensile testing method) leading to a bending stiffness $B=$ $(\pi / 32) E d^{4} \approx 2.3 \times 10^{-6} \mathrm{~J} \mathrm{~m}$. The liquid is a salt-saturated degassed water of mass density $\rho_{\ell}=1.16 \mathrm{~g} / \mathrm{cm}^{3}$ at ambient temperature. The cell is made of three disks of $50 \mathrm{~cm}$ in diameter: a stiff one in 10-mm-thick Duralumin (i), and two successive 15-mm-thick transparent polycarbonate disks (ii) and (iii), allowing observation of the confined rod [Figs. 1(a) and 1(b)]. The two chambers communicate through two holes pierced in disk (ii) and were filled with the liquid through holes pierced in the edges of disks (i) and (iii) in order to equilibrate the pressure in the fluid into the cell. Chamber 2 is sacrificial: when the cell is rotated, the resulting pressure gradient in the liquid bends inwards disk (iii) but the gap is thick enough that it does not close at the maximal frequency of $14 \mathrm{~Hz}$. The pressure is equilibrated between the two faces of disk (ii), allowing the gap to be fixed throughout the whole spacing of chamber 1 . The rod was gently inserted using a hole in the back of disk (i); the thickness of chamber 1 was fixed at $2.5 \mathrm{~mm}$ by a ring of Plexiglas inserted between disks (i) and (ii). The whole setup was placed in a dark room and lit with three stroboscopic lamps with diffusing screens. Movies were taken with a CCD camera. The duration of a flash was short enough $\left(10^{-3} \mathrm{~s}\right)$ to get sharp images of the rod, even at higher velocities. Using a computer interface, the camera and stroboscopes were triggered with the same square periodic signal, while the variator was controlled using a dc voltage. To enhance contrast, we used a white rod on a dark background: an adhesive sheet of black plastic was laid on the Duralumin disk (i). Binary images of the rod were obtained by thresholding. The initial configuration of the rod (Fig. 2) is prepared using eight magnetic beads of diameter $1.8 \mathrm{~mm}$ inserted into the first chamber and moved from outside with a magnet. The cell is then set in rotation; the time taken to reach the desired frequency is approximately $3 \mathrm{~s}$. For each given frequency, a large number of folded configurations is accessible from the same initial configuration (Fig. 2). Indeed, the folding process is nondeterministic and the folded configuration is selected by the experimental noise at the very beginning of the experiment. This experimental noise originates mainly from friction between the rod and the disks and from fluid flow in the cell. During an experiment, the fluid is in solid rotation; the time scale of the transient flow reaching the solid rotation is of the order of $10^{-1} \mathrm{~s}$ which is much smaller than the time needed for the rod to reach equilibrium, as stated below. The second time scale to consider is the lubrication time scale needed to

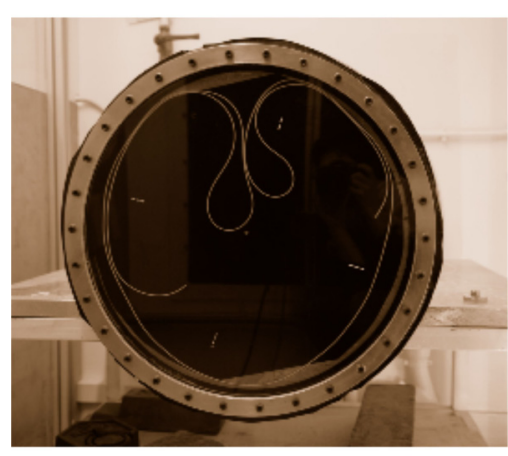

(a)

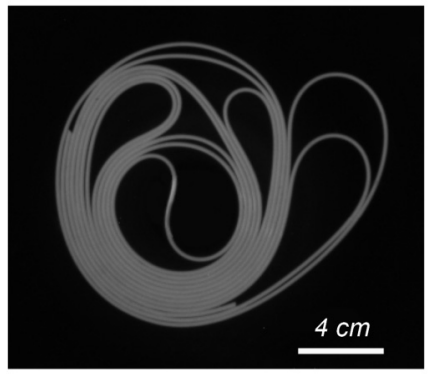

(c)

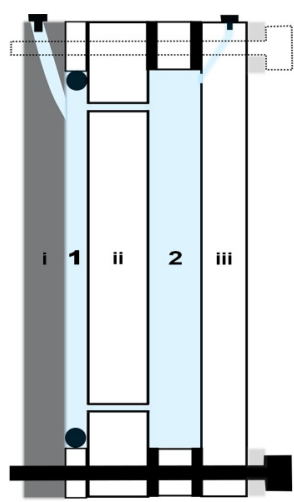

(b)

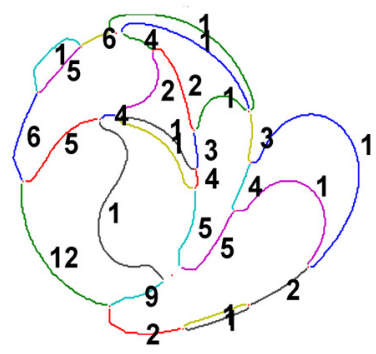

(d)
FIG. 1. (Color online) The experiment. (a) A rod of density $1 \mathrm{~g} / \mathrm{cm}^{3}$ is confined in a circular Hele-Shaw cell, filled with saltsaturated water of density $1.16 \mathrm{~g} / \mathrm{cm}^{3}$. (b) The cell is made of a superposition of three disks; the intermediate one (ii) is pierced with two holes to enable equilibration of pressure into the liquid. This setup enables the fixing of the thickness of chamber 1 where the rod is placed, chamber 2 being a sacrificial room. The chambers are made watertight with flat and toric joints shown in black. (c) Example of an equilibrium configuration obtained when a centripetal force is generated by the rotation of the cell around its axis. (d) Corresponding skeletonized image in which the number of layers per branch is defined. Figure reproduced from [33].

expel fluid between two segments of rod, which is of the order of $1 \mathrm{~s}$, again much smaller than the rod equilibration time scale.

First, the resulting configurations can be characterized using their radius of gyration,

$$
R_{g}=\sqrt{\frac{1}{L} \int_{0}^{L} r^{2}(s) d s},
$$

where $s$ is the curvilinear coordinate along the rod and $r(s)$ the distance to the cell axis of rotation. This quantity is directly calculated using the binary image of the confined rod. The radius of gyration decreases with time (Fig. 3), rapidly when the rotation is started and then more slowly, reaching a plateau after a time lapse of the order of $10^{3} \mathrm{~s}$. This final value is not unique and differs according to the realizations at a given frequency. As we investigated only equilibrium configurations, we waited $1800 \mathrm{~s}$ for each value of the frequency before taking measurements, to ensure that equilibrium is reached. When averaging over realizations, the mean radius of gyration $\bar{R}$ is found to be a decreasing function of confinement strength (inset in Fig. 3). To assess this result, we compare the observed 

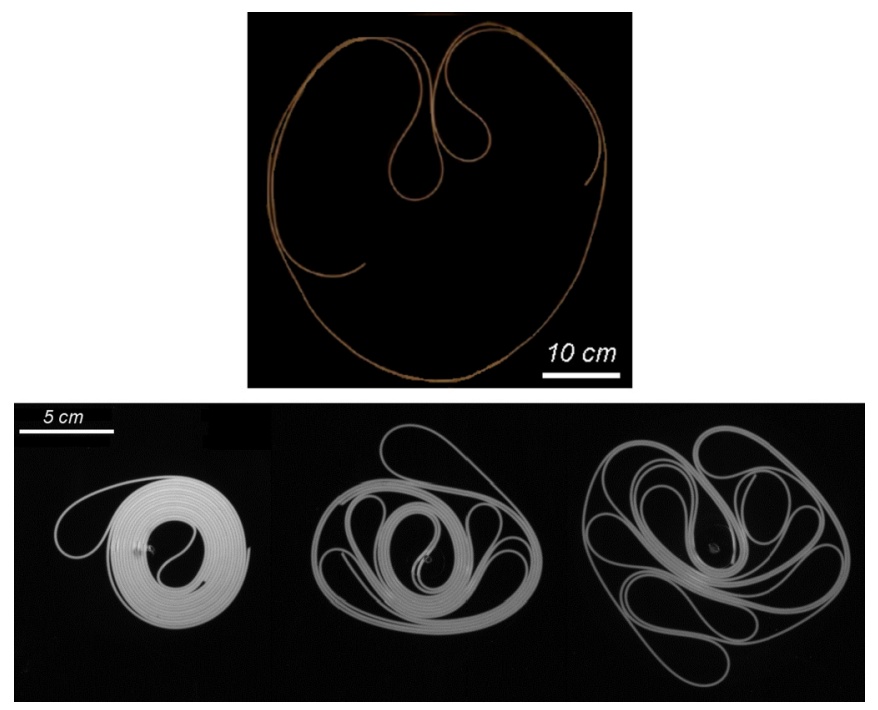

FIG. 2. (Color online) Top picture: the initial configuration of the rod. Bottom pictures: three different folded configurations resulting from experiments with the same parameters, where the initially still cell is set into rotation to a velocity of $14 \mathrm{~Hz}$. A wide variety of geometries are obtained from the same initial configuration: the folding process is nondeterministic.

configuration with the optimal configuration, namely, the Archimedean spiral that is the more compact configuration (see Fig. 9). By minimizing the energy of the spiral versus the radius of its empty core, we find that its radius of gyration $R_{g}^{(s)}$ is given by Eqs. (A11) and (A12) in the Appendix. Values are

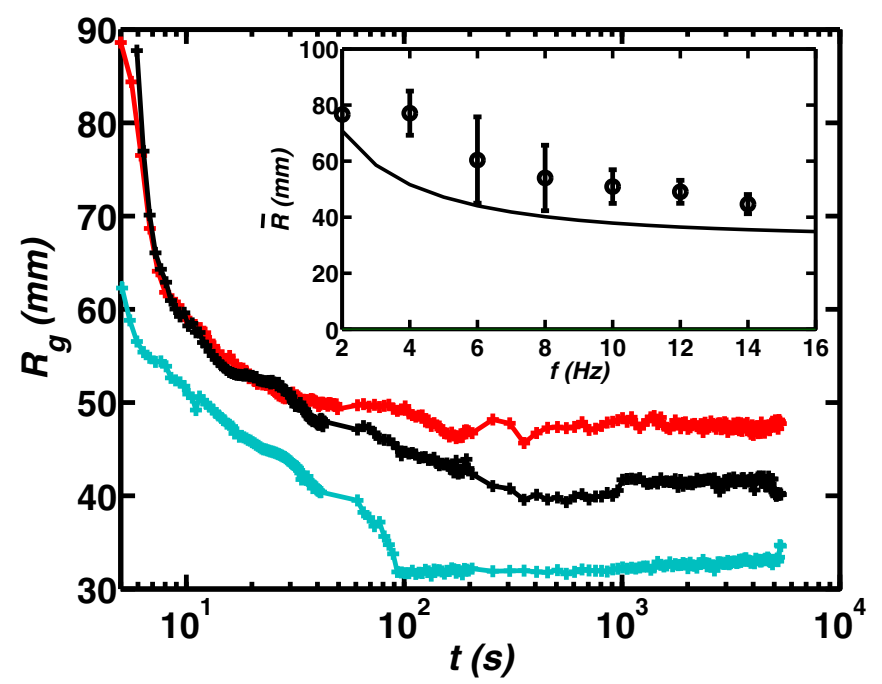

FIG. 3. (Color online) The radius of gyration $R_{g}$ as a function of time for three realizations in which the cell was set into rotation from 0 to $14 \mathrm{~Hz}$ in $3 \mathrm{~s}$. Radii reach a plateau value in about $10^{3} \mathrm{~s}$. The final radius differs according to the realization. On average (inset), the radius of gyration $\bar{R}$ (०) decreases with the strength of confinement (quantified by the rotation frequency $f$ of the disk). The bars represent the widths of the distribution for each frequency of rotation, measured on the whole set of experiments run for this frequency. Measurements are compared to the radius of gyration of a spiral (-) obtained for the same confinement intensity [Eqs. (A11) and (A12)]. reported in Fig. 3 as a function of the rotation velocity. For each confinement intensity, $R_{g}^{(s)}$ gives a lower bound for the measured $\bar{R}$ because experimental geometries rarely reach the optimal folding state. Indeed, when the experiment is repeated with the same control parameters, a large diversity of sizes and geometries is observed, all of which are always less compact than the optimal spiral.

To go beyond this statement, we need measures of the geometrical and mechanical properties of equilibrium patterns. To do this, the skeleton of folded configurations is extracted from binary images. Voids of area smaller than a given threshold have been filled, but this thresholding process did not influence final results. Vertices are detected as self-contact points, i.e., points of the skeleton having three neighbors [Figs. 1(c) and 1(d)]. We define branches as portions delimited by two vertices. A given branch may contain several layers of the rod: the thickness of the branch on the binary image directly yields the number of layers. The analysis of the experimental patterns was automated in MATLAB, allowing us to treat a large amount of data. Having access to the geometry of the confined rod, we are able to quantify the curvature, length, and energy of branches and to perform statistics over experimental realizations. As we provide the same measurements as in Ref. [29], we will compare the results of the two experimental situations.

\section{STATISTICAL DISTRIBUTIONS}

In this section, we report on the probability distribution functions (PDFs) of the geometrical and mechanical properties of the folded configurations. The PDFs $\rho(x)$ associated with the variable $x$ are grouped using two types of representation. For the geometrical quantities (curvature and length), the PDFs are performed over realizations whose radii of gyration satisfy $R_{g}=\bar{R} \pm \Delta R_{g}$ with a value of $\Delta R_{g}=5 \mathrm{~mm}$ chosen to ensure convergence of the statistics. For the mechanical quantities (bending and total energies), each PDF contains configurations with the same strength of confinement, i.e., the same rotation frequency $f$. Indeed, the radius of gyration characterizes the geometry while the confinement strength sets the energy scale. The error bars $\delta \rho$ of the experimental PDFs are estimated using the standard deviation $\delta \rho=\rho / \sqrt{n}$ of the corresponding histograms $n(x)$. In order to increase the set of data, we considered two types of experimental configuration. The first type of configuration is obtained following the same protocol as described above: the cell is set into rotation from $0 \mathrm{~Hz}$ until the desired frequency $f$ and the equilibrium configuration is assumed to be reached after 1800 s. Configurations of the second type are obtained from an initially folded configuration, by increasing or decreasing the frequency to the desired frequency of rotation. The delay of $1800 \mathrm{~s}$ between two observations is always respected. We checked that the resulting PDFs are not affected by the choice of the initial configuration. The statistical data analysis has been performed over 186 configurations obtained from 56 experimental realizations. The number of branches for each configuration varied from 12 to 67 , with an average of 32 branches. The total number of branches for each set is of the order of $10^{3}$, which allows for accurate statistics. 
(a)
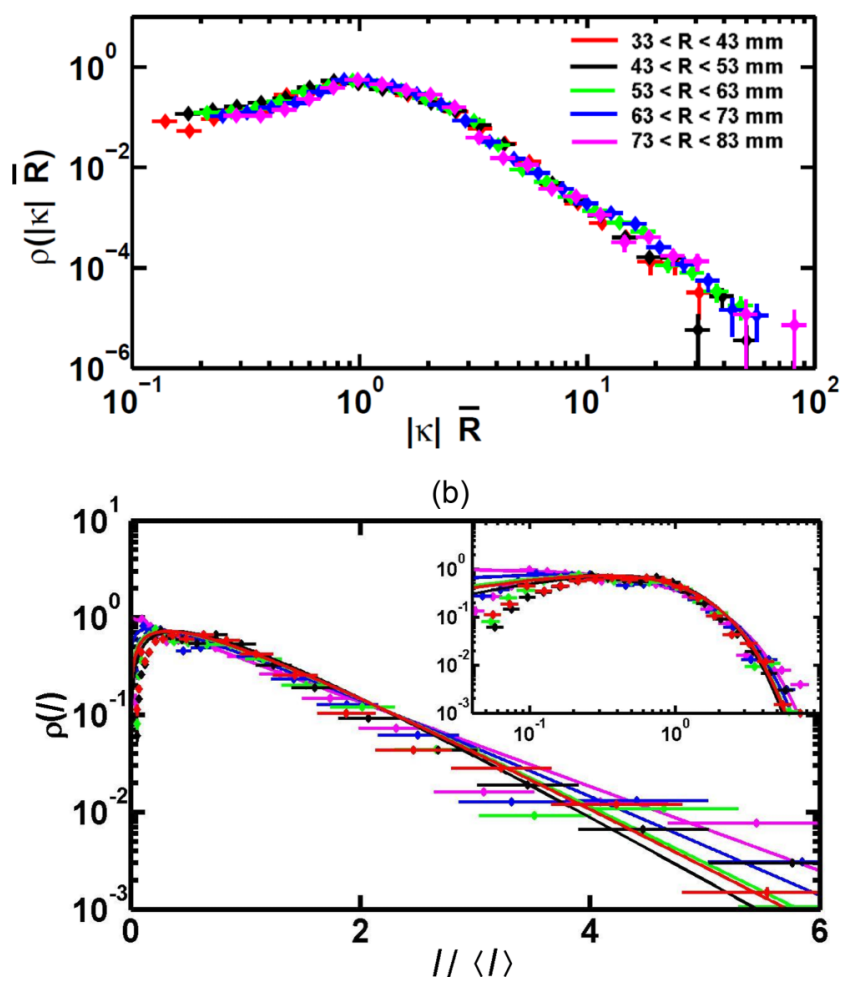

FIG. 4. (Color online) Statistics of the geometrical properties for six different sets; each set includes measurements of folded configurations that have a given interval of radii of gyration indicated in the legend. (a) Experimental PDFs $\rho(\kappa \bar{R})$ of the mean curvature of branches $\kappa$ normalized by the average radius of gyration of the group $\bar{R}$. (b) Experimental PDFs $\rho(l /\langle l\rangle)$ of the length of branches $l$ normalized by the mean branch length $\langle l\rangle$ of each set. Gamma distributions $f_{\Gamma}^{\alpha, \chi}(l)$, Eq. (2), with the same mean and variance as experimental data are represented. Inset: zoom on the smallest lengths of branches.

Curvature. Figure 4(a) shows the PDFs of the mean curvature of branches normalized by the radius of gyration of the configurations. The mean curvature is defined as the average of the absolute value of the curvature at each point of the branch. The distributions have the same shape independently of the intensity of confinement. They are peaked around the radius of gyration and show a decreasing exponential tail. In other words, the most probable radius of curvature of branches is the radius of gyration. Compared to the results of Refs. [29,30], Fig. 4(a) is similar to the PDF of branches that have a curvature partially imposed by the confining solid walls. This property will be discussed below.

Length. PDFs of the lengths of branches are plotted in Fig. 4(b) and are rather well described by a Gamma distribution function defined by

$$
f_{\Gamma}^{\alpha, \chi}(x)=\frac{(x / \chi)^{\alpha}}{\Gamma(\alpha) x} \exp (-x / \chi)
$$

where $\Gamma$ is Euler's Gamma function and

$$
\alpha=\frac{\langle x\rangle^{2}}{\left\langle x^{2}\right\rangle-\langle x\rangle^{2}}, \quad \chi=\alpha^{-1}\langle x\rangle,
$$

allowing for a fit without any adjustable parameters. Figure 4(b) also shows that the width of the distribution decreases when the confinement is increased. In Sec. IV A, the behavior of the exponent $\alpha$ as function of the strength of the confinement will be detailed. Note that in the experimental configuration of Refs. [29,30], PDFs of the branch lengths were described by a decreasing exponential only. Such exponential distributions have also been observed for fold lengths in numerical simulations of crumpled sheets [21]. We believe that the difference between the present study and previous ones is due to plastic and friction effects that are inherent in the experiments of Refs. [29,30] and that induce lower cutoffs in the lengths of the branches, preventing their division into smaller ones, whereas the folds in [21] also seem to be longer than a cutoff.

Energy. The geometrical characterization of the folded configuration allows measurement of its mechanical properties through the computation of the energies involved. The total energy of a branch is the sum of two terms, $E_{\text {tot }}=E_{b}+E_{\text {conf }}$, with $E_{b}$ the elastic bending energy:

$$
E_{b}=N \frac{B}{2} \int_{0}^{l} \kappa^{2}(s) d s
$$

where $\kappa(s)$ is the local curvature of the branch, $l$ its length, and $N$ the number of layers of the corresponding branch. The second term $E_{\text {conf }}$ is the confinement energy induced by the rotation of the cell and is given by

$$
E_{\mathrm{conf}}=N \frac{\pi}{8}\left(\rho_{l}-\rho_{r}\right) d^{2} \omega^{2} \int_{0}^{l} r^{2}(s) d s .
$$

Figure 5 shows that the PDFs are weakly dependent on the strength of confinement and can be described by two power laws, valid for both bending and total energy distributions, of exponents $-0.3 \pm 0.1$ (over four decades) for small energies and $-2.3 \pm 0.3$ (over two decades) for higher energies. For both bending and total energies, the transition between these two scaling behaviours is sharp and yields characteristic energies $E_{b}^{t}$ and $E_{\text {tot }}^{t}$ that suggest the existence of an energy scale for the folding process. This will be discussed in Sec. IV B.

At this stage, a question that arises naturally is how the present results compare with those of the experiments reported in $[29,30]$ where the energy distributions were described by Gamma laws. We argue that the results of the two experimental configurations agree qualitatively. The difference in the distributions could be related to the experimental setups generating different stacking process. Nevertheless, the advantage of the present experiment is that it allows a wide range of energy scales to be spanned (six decades) and higher energies to be reached. This is not the case of the previous experimental setup: the narrow range of the exponential tail in the results of the energy distribution might be ascribed to a crossover between two power law behaviors. Another possible reason might be the difference in the dissipation mechanisms, friction between layers in the previous experiment and viscous dissipation through the fluid motion in the current one, leading the two systems to scan differently available possible configurations.

In the following and on the basis of the results presented above, we show how configurations become relatively more ordered upon increasing the confinement and highlight the 

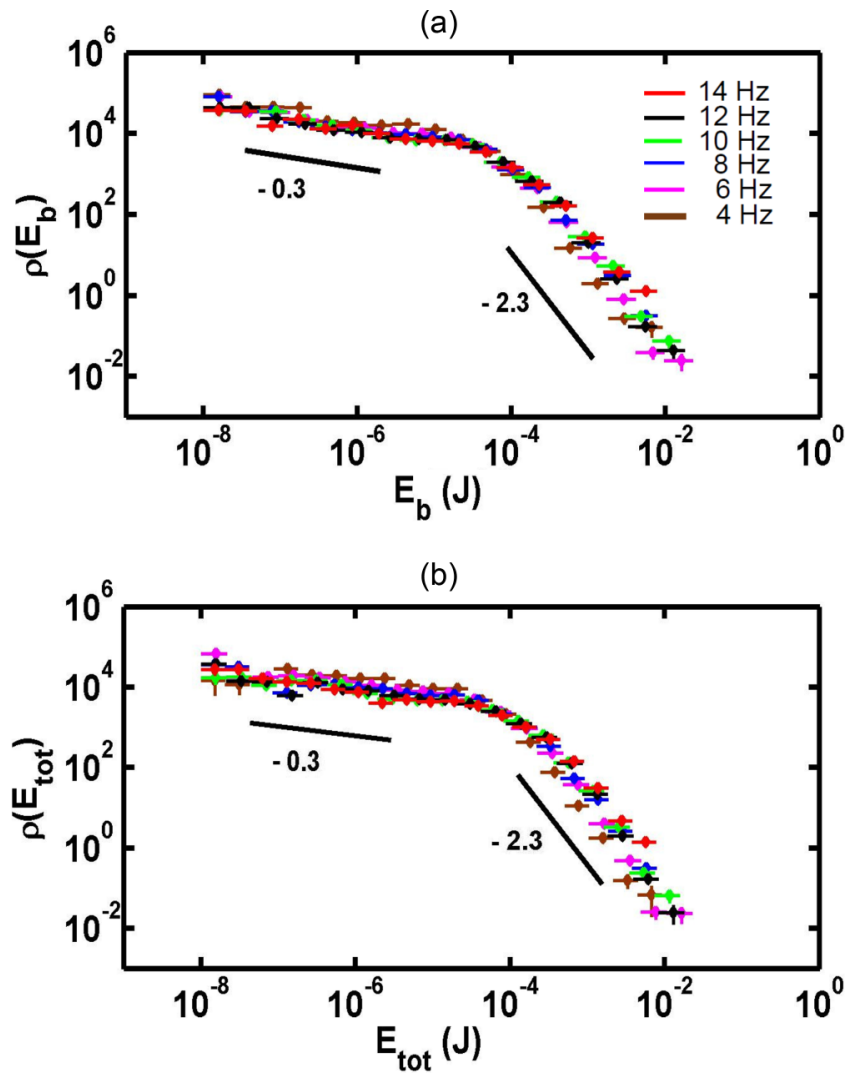

FIG. 5. (Color online) Statistics of the energy for sets of data obtained at six different frequencies $f$. (a) Experimental PDFs $\rho\left(E_{b}\right)$ of the bending energy of branches $E_{b}$ [see Eq. (4)]. (b) Experimental PDFs $\rho\left(E_{\text {tot }}\right)$ of the total energy of branches $E_{\text {tot }}$, including bending and confinement terms [see Eq. (5)]. Note that the distributions are not normalized and energies are given in joules.

existence of an energy scale that could be a relevant quantity for a thermodynamic description of folded elastic objects.

\section{DISCUSSION}

\section{A. Stacking process}

The stacking process is the mechanism by which a nematic order occurs $[22,30,34]$. In the present experiment, it refers to the collapse of different parts of the rod in the same state defined by the same geometrical and mechanical properties. In other words, when the confinement increases, new layers are added to existing branches, or different branches can even merge to form a thicker one. The Gamma distribution of branch lengths shown in Fig. 4(b) can be explained in the framework of this stacking process [17]. If the sizes of layers are independent, their length distributions result from a random splitting of the rod. This would lead to an exponential distribution of sizes [35]. In the present experiment, both the number of layers included in the same branch, $N_{l / b}$, and the lengths of the branches are distributed. The length of branches is determined effectively by the averaging of all the lengths of layers included in this branch. Accordingly, we expect a Gamma distribution of parameter $\alpha$ [defined in (2)] equal to the number of averaged random variables with exponential distributions, that is, $\alpha=\left\langle N_{l / b}\right\rangle$.

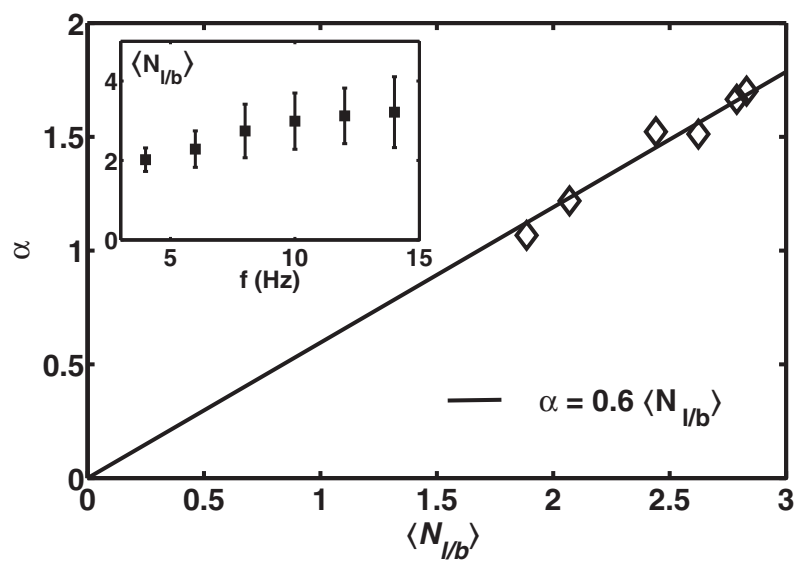

FIG. 6. The parameter $\alpha$ of the Gamma distribution $\rho(l /\langle l\rangle)$ as defined by Eq. (2), as a function of the average number of superposed layers per branch $\left\langle N_{l / b}\right\rangle$. Inset: $\left\langle N_{l / b}\right\rangle$ as a function of the frequency of rotation.

The parameter $\alpha$ can be determined from experimental Gamma distributions using the average and standard deviation of the data. It is found that it increases with the frequency of rotation $f$. On the other hand, image analysis allows measurement of the mean number of layers per branch, $\left\langle N_{l / b}\right\rangle$. For a given frequency, it is determined by counting the mean number of layers per branch averaged over all branches of a given configuration and then by averaging the results over all the configurations. Figure 6 shows that $\left\langle N_{l / b}\right\rangle$ increases with confinement and is related to $\alpha$ through

$$
\alpha=a\left\langle N_{l / b}\right\rangle,
$$

where $a=0.6 \pm 0.06$. This linear behavior with a proportionality constant of order 1 confirms the scenario of a stacking process with an order parameter given by the average number of layers or equivalently by the mean length of branches.

Using a different approach, the mean number of layers in the same geometrical state, $\left\langle N_{l / b}\right\rangle$, was identified as an order parameter and the spiral, for which all layers are superposed, was shown to be the ideally stacked configuration [33]. Figure 6 shows that $\left\langle N_{l / b}\right\rangle$ increases with $f$ and thus the folded rod becomes more ordered, through a stacking process, when confinement is increased. The behavior shown in Fig. 4(a) can be explained using these results. Stacking results in the formation of thicker branches that stiffen the structure. As a result, the folded rod is localized around the radius of gyration of the configuration and the remaining branches behave as if they were in contact with a virtual container of radius $R_{g}$. Consequently, the PDFs of branch curvature are always peaked around $R_{g}$. Whereas the present setup was built in order to fold a rod in the absence of a rigid container and to avoid sampling problems encountered in $[29,30]$, the stacking mechanism is found to impose geometrical constraints on the folding process. We note that the authors of [29] had to consider two subsystems in thermal equilibrium, while the present setup allows us to consider only a single system, which is simpler to analyze.

\section{B. An energy scale}

The PDFs of bending and total energies show similar behaviors characterized by two quasi-power-laws at small 

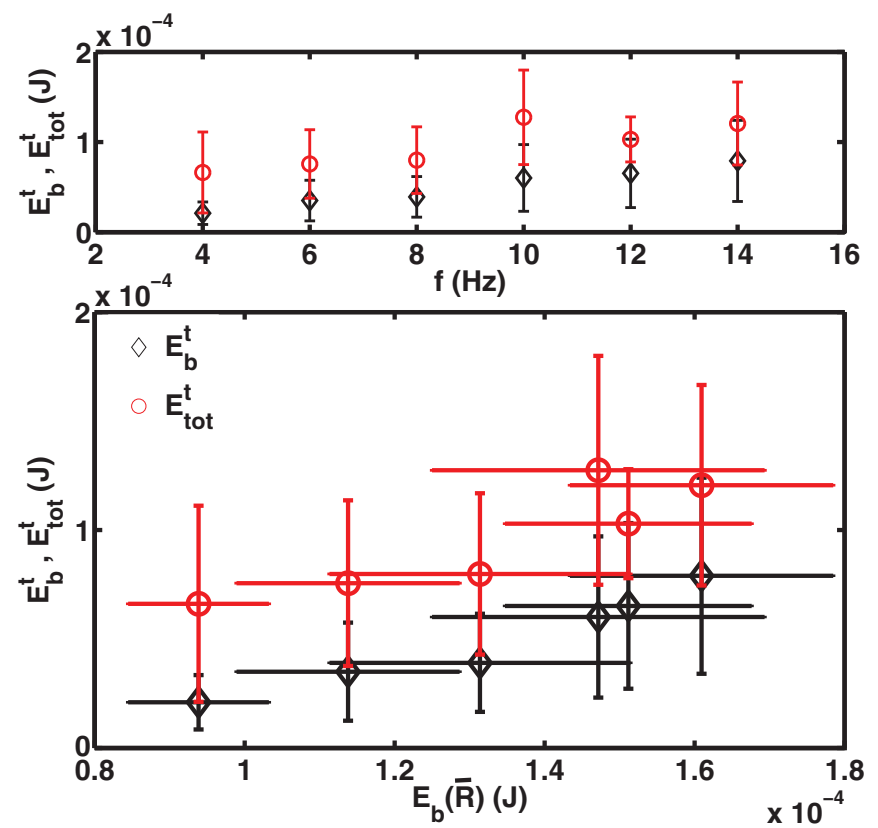

FIG. 7. (Color online) The energy scales $E_{b}^{t}(\diamond)$ and $E_{\text {tot }}^{t}(\circ)$ as functions of the rotation frequency $f$ (top) and as functions of the bending energy of a ring of radius $\bar{R}(f)$ given by Eq. (7) (bottom).

and high energies. The energy scales $E_{b}^{t}$ and $E_{\text {tot }}^{t}$ at the transition between the two behaviors can be determined from the experimental PDFs of the bending and total energies. The bending $\left(E_{b}^{t}\right)$ and total $\left(E_{\text {tot }}^{t}\right)$ energies at which the transition occurs as a function of the confinement strength are shown in Fig. 7. Despite large error bars, the dependence on the frequency of rotation shows that both $E_{b}^{t}$ and $E_{\text {tot }}^{t}$ increase with the confinement. The analysis of the geometrical properties of the folded configurations reveals a stacking process around a virtual container of radius $\bar{R}(f)$ that is larger than the radius of gyration of the spiral with the same confinement intensity (see Sec. II). Therefore, a natural choice of energy scale to compare with is the bending energy of a rod spooled around the position given by the mean radius of gyration of the corresponding configuration. As a first approximation, this virtual container can be modeled by a ring of radius $\bar{R}(f)$ with bending energy given by

$$
E_{b}(\bar{R})=\frac{\pi B}{\bar{R}} .
$$

Figure 7 shows that $E_{b}^{t}$ and $E_{\text {tot }}^{t}$ are of the same order of magnitude as $E_{b}(\bar{R})$, although they are slightly smaller, and behave similarly: they increase when the confinement strength is increased. This suggests the existence of a scale of energy that is imposed by the stacking process. It is reflected in the two different behaviors (Fig. 5), depending on whether the energies are smaller or larger than the energy scale.

\section{SUMMARY AND CONCLUSION}

To summarize, the PDFs of the geometrical quantities of configuration, length, and curvature support the existence of a stacking process. This process has also been evidenced in the analysis of energy distributions. Moreover, the stacking is reinforced when the confinement is increased, which enhances the geometrical order of patterns formed by the folded rod. Although it is not achieved in experiments, the most ordered configuration is the spiral; it is also the ground state in terms of energy, while it allows minimization of both the curvature and the size of the system. If layers are stacked together when confined, the configurations become closer to the spiral, even though geometrical constraints prevent it. Therefore, our first main result is that, when confined, the system tends to become closer to the ground state. Our second result comes from the observation of energy PDFs. The stacking process governs the distribution of the branchs energy, imposing a scale which is linked to the bending energy of the spiral. The size of the spiral depends on the confinement intensity, and thus the energy scale follows the same dependance.

We interpret these two results as follows: selected patterns of the folded rod are excited states of a unique ground state, the spiral. For a given confinement strength, a ring having the mean size $\bar{R}$ at this confinement imposes the energy distribution throughout the system. This can be interpreted as that, for a given injected energy, all available configurations of the phase space are excited states of the ground state. Because the stacking of layers increases with the confinement, we claim that the system tends to become closer to the ground state when the available energy is increased. However, because of self-avoidance, the system is topologically frustrated and reaches only local minimal energy states, rarely finding its route through the spiral configuration. This scenario is schematically summarized in Fig. 8.

To conclude, we built an experiment allowing the twodimensional confinement of a rod in a parabolic potential. This parabolic confinement differs from the rigid confinement of [16,28-30], allowing us to explore the role of the type of confinement and to address more comprehensively our initial questions. What governs the pattern geometry? A stacking process promotes the formation of thick branches and imposes the geometrical characteristics of the patterns:

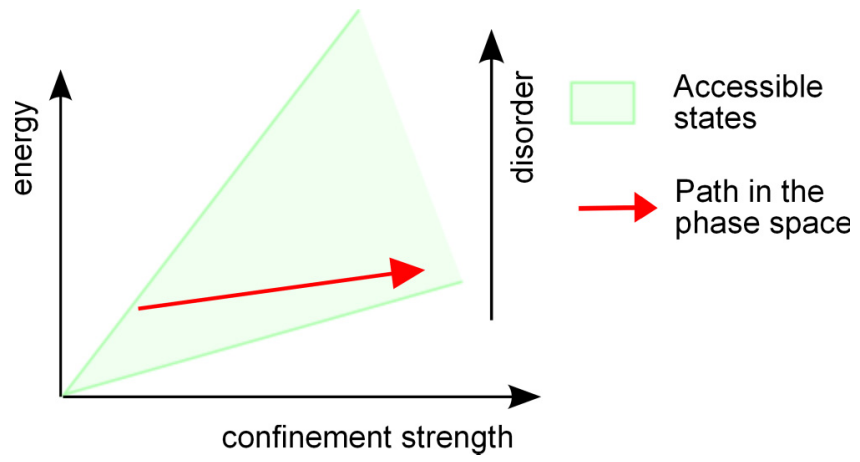

FIG. 8. (Color online) Phase space of folded configurations accessible by the experimental setup shown in Fig. 1. The lower limit corresponds to the energy of the spiral, which is the most ordered and least energetic configuration. The upper limit of the accessible configurations is not clearly defined. The arrow represents the selected configurations as observed during the experiments. Increasing confinement strength makes the pattern more ordered, and while the global energy of the available configurations increases, the energy scale selected becomes closer to the ground state. 
the curvature and length distributions. Our second goal was to propose a description of the confined states as an ensemble of excited states of the spiral, the ground state in terms of order and energy. The ground state imposes an energy scale, which governs the partition of energy throughout the system. When the whole available energy is increased by external injection, a relaxation of the system towards the fundamental state occurs through a stacking process. However, this global energy minimum is rarely achieved, because of the topological frustration induced by the constraint of self-avoidance. Finally, the energy scale extracted from our experimental results could be a useful tool to define an effective temperature that measures the internal order and from which a thermodynamical theory of these confined objects can be built.

\section{ACKNOWLEDGMENTS}

The authors are grateful to L. Boué, F. Corson, S. Deboeuf, and E. Katzav for enlightening discussions and contributions to earlier discussions of this work.

\section{APPENDIX: THE RADIUS OF GYRATION OF A CONFINED SPIRAL}

The spiral configuration of Fig. 9 minimizes the curvature, stacking all the parts of the rod together, and minimizes the size. In the case of an opened rod, of diameter $d$, length $L$, and bending stiffness $B$, the spiral presents a central void, with a radius $R_{c}$ which evolves with the confinement intensity. In the case of a rod of mass density $\rho_{r}$, placed in a liquid of mass density $\rho_{l}>\rho_{r}$, and confined by a centripetal force generated by a rotation of angular velocity $\omega=2 \pi f$, we calculate the internal radius $R_{c}$ and then the radius of gyration $R_{g}^{(s)}$ of the spiral as a function of the confinement intensity, i.e., the rotation frequency of the cell. The spiral radius parametrized by the angle $\theta$ is

$$
\rho(\theta)=\frac{d}{2 \pi} \theta+R_{c}
$$

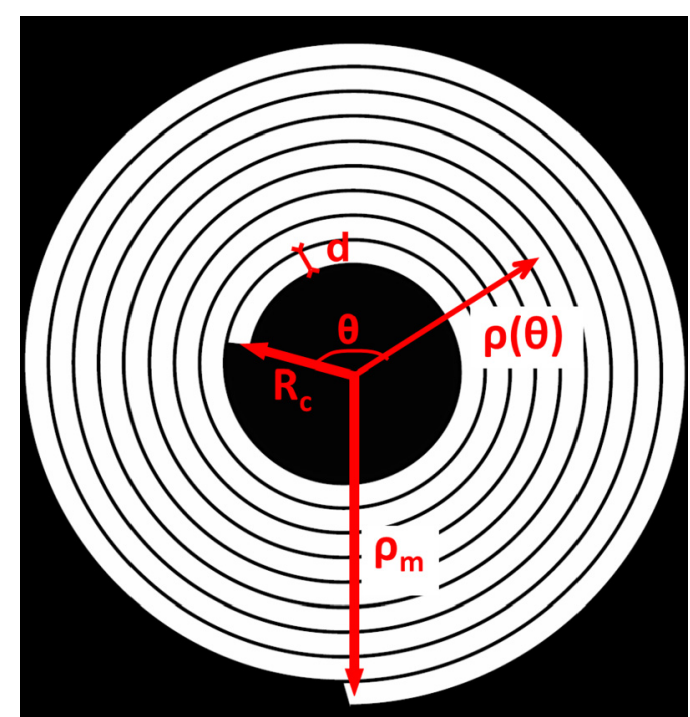

FIG. 9. (Color online) Sketch of an Archimedean spiral of internal radius $R_{c}$ and separation distance $d$.
The arc length coordinate of the spiral $s(\theta)$ is defined by $d s=$ $\rho d \theta$, with $s(0)=0$ and $s\left(\theta_{\max }\right)=L$. Therefore, the maximal angle $\theta_{\max }$ depends on the total length of the $\operatorname{rod} L$ through

$$
L=\int_{0}^{\theta_{\max }} \rho d \theta .
$$

Using (A1) and (A2), the maximal angle $\theta_{\max }$ is given by

$$
\theta_{\max }=\frac{2 \pi R_{c}}{d}(\sqrt{1+\kappa}-1)
$$

where

$$
\kappa=\frac{d L}{\pi R_{c}^{2}}
$$

is a geometrical parameter that compares the total area of the spiral and the central void area. The maximal radius of the spiral is then

$$
\rho_{\max }=R_{c} \sqrt{1+\kappa} \text {. }
$$

The confinement energy $E_{\text {conf }}$ and the bending energy $E_{b}$ are given by

$$
\begin{gathered}
E_{\text {conf }}=\frac{1}{2} \omega^{2}\left(\rho_{l}-\rho_{r}\right) \pi\left(\frac{d}{2}\right)^{2} \int_{0}^{\theta_{\max }} \rho^{3} d \theta, \\
E_{b}=\frac{B}{2} \int_{0}^{\theta_{\max }} \frac{d \theta}{\rho},
\end{gathered}
$$

or, after integration,

$$
\begin{gathered}
E_{\mathrm{conf}}=-\omega^{2}\left(\rho_{l}-\rho_{r}\right) \frac{\pi^{2} d}{16} R_{c}^{4}\left[(1+\kappa)^{2}-1\right], \\
E_{b}=\frac{\pi B}{2 d} \ln (1+\kappa) .
\end{gathered}
$$

The internal radius $R_{c}$ minimizes the total energy of the spiral. Solving the equation $d\left(E_{b}+E_{\text {conf }}\right) / d R_{c}=0$, one finds that $R_{c}$ is a solution of the polynomial equation

$$
R_{c}^{4}+\frac{d L}{\pi} R_{c}^{2}-\frac{B}{\omega^{2}\left(\rho_{l}-\rho_{r}\right) \pi\left(\frac{d}{2}\right)^{2}}=0 .
$$

The positive root of this equation gives

$$
R_{c}^{2}=\frac{d L}{2 \pi}\left[\sqrt{1+\frac{\pi^{2} E}{2 \omega^{2}\left(\rho_{l}-\rho_{r}\right) L^{2}}}-1\right],
$$

where the definition of the bending rigidity $B=(\pi / 32) E d^{4}$ has been used. Finally, the radius of gyration of the spiral, $R_{g}^{(s)}=\sqrt{1 / L \int_{0}^{L} \rho^{2}(s) d s}$, is given by

$$
R_{g}^{(s)}=R_{c} \sqrt{1+\frac{d L}{2 \pi R_{c}^{2}}} .
$$


[1] P. de Gennes and J. Prost, The Physics of Liquid Crystals (Oxford University Press, Oxford, 1974).

[2] J. Arsuaga, M. Vazquez, S. Trigueros, D. Sumners, and J. Roca, Proc. Natl. Acad. Sci. USA 99, 5373 (2002).

[3] E. Katzav, M. Adda-Bedia, and A. Boudaoud, Proc. Natl. Acad. Sci. USA 103, 18900 (2006).

[4] H. Kobayashi, B. Kresling, and J. F. V. Vincent, Proc. R. Soc. London, Ser. B 265, 147 (1998).

[5] E. Couturier, S. C. du Pont, and S. Douady, PLoS ONE 4, e7968 (2009).

[6] J. A. Rogers, T. Someya, and Y. G. Huang, Science 327, 1603 (2010).

[7] R. J. Wootton, J. Zool. 193, 447 (1981).

[8] T. A. Witten and H. Li, Europhys. Lett. 23, 51 (1993).

[9] A. E. Lobkovsky, S. Gentes, H. Li, D. Morse, and T. A. Witten, Science 270, 1482 (1995).

[10] A. E. Lobkovsky, Phys. Rev. E 53, 3750 (1996).

[11] M. Ben Amar and Y. Pomeau, Proc. R. Soc. London, Ser. A 453, 729 (1997).

[12] E. Cerda, S. Chaieb, F. Melo, and L. Mahadevan, Nature (London) 401, 46 (1999).

[13] T. Mora and A. Boudaoud, Europhys. Lett. 59, 41 (2002).

[14] T. Liang and T. A. Witten, Phys. Rev. E 71, 016612 (2005).

[15] S. Deboeuf, E. Katzav, A. Boudaoud, D. Bonn, and M. AddaBedia, Phys. Rev. Lett. 110, 104301 (2013).

[16] L. Boué, M. Adda-Bedia, A. Boudaoud, D. Cassani, Y. Couder, A. Eddi, and M. Trejo, Phys. Rev. Lett. 97, 166104 (2006).

[17] E. Sultan and A. Boudaoud, Phys. Rev. Lett. 96, 136103 (2006).

[18] F. Plouraboué and S. Roux, Physica A 227, 173 (1996).
[19] D. L. Blair and A. Kudrolli, Phys. Rev. Lett. 94, 166107 (2005).

[20] C. A. Andresen, A. Hansen, and J. Schmittbuhl, Phys. Rev. E 76, 026108 (2007).

[21] G. A. Vliegenthart and G. Gompper, Nat. Mater. 5, 216 (2006).

[22] Y. C. Lin, J. M. Sun, J. H. Hsiao, Y. Hwu, C. L. Wang, and T. M. Hong, Phys. Rev. Lett. 103, 263902 (2009).

[23] H. Aharoni and E. Sharon, Nat. Mater. 9, 993 (2010).

[24] A. D. Cambou and N. Menon, Proc. Natl. Acad. Sci. USA 108, 14741 (2011).

[25] K. Matan, R. B. Williams, T. A. Witten, and S. R. Nagel, Phys. Rev. Lett. 88, 076101 (2002).

[26] B. Thiria and M. Adda-Bedia, Phys. Rev. Lett. 107, 025506 (2011).

[27] S. F. Edwards and R. B. S. Oakeshott, Physica A 157, 1080 (1989).

[28] A. S. Balankin and O. S. Huerta, Phys. Rev. E 77, 051124 (2008).

[29] S. Deboeuf, M. Adda-Bedia, and A. Boudaoud, Europhys. Lett. 85, 24002 (2009).

[30] M. Adda-Bedia, A. Boudaoud, L. Boué, and S. Deboeuf, J. Stat. Mech.: Theory Expt. (2010) P11027.

[31] N. Stoop, J. Najafi, F. K. Wittel, M. Habibi, and H. J. Herrmann, Phys. Rev. Lett. 106, 214102 (2011).

[32] J. Najafi, N. Stoop, F. Wittel, and M. Habibi, Phys. Rev. E 85, 061108 (2012)

[33] E. Bayart, S. Deboeuf, F. Corson, A. Boudaoud, and M. AddaBedia, Europhys. Lett. 95, 34002 (2011).

[34] D. Aristoff and C. Radin, Europhys. Lett. 91, 56003 (2010).

[35] W. Feller, An Introduction to Probability Theory and Its Applications (John Wiley \& Sons, New York, 1970). 